DOI: https://doi.org/10.46296/yc.v5i9ucedespsoct.0122

\title{
PROPORCIÓN ADECUADA DE SALES FUNDENTES PARA ALCANZAR LA TEXTURA REQUERIDA DE QUESO FUNDIDO UNTABLE
}

\section{AN ADEQUATE PROPORTION OF FLUX SALTS TO ACHIEVE THE REQUIRE TEXTURE IN SPREADABLE MELTED CHEESE}

\author{
Díaz-Sánchez Jorge ${ }^{1}$; García-González Mercedes Caridad 2; Lorente-González \\ Gustavo Yasser ${ }^{3}$; Cardoso-Paneque Lianet ${ }^{4}$ \\ ${ }^{1}$ Profesor Asistente de la Universidad de Camagüey "Ignacio Agramonte Loynaz". \\ Camagüey, Cuba. Correo: jorged.sanchez@reduc.edu.cu. ORCID ID: \\ https://orcid.org/0000-0003-1193-8873
}

2 Profesor Titular de la Universidad de Camagüey "Ignacio Agramonte Loynaz". Camagüey, Cuba. Correo: mercedes.garcia@reduc.edu.cu. ORCID ID: http://orcid.org/0000-0003-4785-8605

${ }^{3}$ Investigador Agregado en la Universidad de Ciego de Ávila Máximo Gómez Báez. Ciego de Ávila, Cuba. ORCID ID: http://orcid.org/0000-0003-1008-4079

${ }^{4}$ Estudiante de quinto año de Licenciatura en Ciencias Alimentarias de la Universidad de Camagüey "Ignacio Agramonte Loynaz". Camagüey, Cuba. Correo:

lianet.cardoso@reduc.edu.cu.

\begin{abstract}
Resumen
El queso procesado o fundido se ha convertido en una buena alternativa para las industrias lácteas, debido a que tiene un período de vida útil prolongado y presenta mejor facilidad de manejo en las empresas. El objetivo de la investigación fue establecer una proporción adecuada de sales fundentes que permita alcanzar la textura requerida en el queso fundido untable, mediante una mezcla de sales de bifosfato y monocitrato de sodio. La investigación fue realizada mediante un diseño de mezcla Simplex-Lattice con dos componentes (monocitrato sódico y bifosfato sódico) y tres variables respuestas ( $\mathrm{pH}$, humedad y textura), para la cual se llevaron a cabo 15 corridas experimentales. Los resultados obtenidos en la variable $\mathrm{pH}$ indicaron buena correlación ya que los valores son elevados, las variables humedad y textura mostraron que no existe correlación con respecto a las sales. Se obtuvieron valores de humedad de 59,36 a 65,69 $\%$ y la textura de 11,86 a 20,0 siendo la media de los valores de textura caracterizados como bueno. Atendiendo a los resultados alcanzados en la optimización múltiple se obtuvo que la mejor formulación de sales fundentes para queso fundido untable es: $70 \%$ de bifosfato de sodio y 30 $\%$ de monocitrato de sodio, o emplear el $3 \%$ de la mezcla solo con bifosfato de sodio ya que ambas formulaciones ofrecen como resultado la textura deseada.
\end{abstract}

Palabras claves: queso fundido untable, sales fundentes, sales de bifosfato y monocitrato de sodio.

\begin{abstract}
Processed or melted cheese is turned into a good alternative to dairy industry, due to its long period of duration and it offers better facilities in management of the industries. The objective of the researching was to establish an adequate proportion of fluxing salts to acquire required texture in spreadable melted cheese by means of a mixture of sodium biphosphate salts and sodium monositrate. The researching was carried out by means of a design of a mixture Simplex-Lattice with two components (sodium monositrate and biphosphate salts) and three "variable answers" $(\mathrm{pH}$, humidity and texture), 15 experimental tests were applied. The results obtained in $\mathrm{pH}$
\end{abstract}

Información del manuscrito:

Fecha de recepción: 26 de julio de 2021.

Fecha de aceptación: 29 de septiembre de 2021.

Fecha de publicación: 11 de octubre de 2021. 
variable indicated good correlation since values are high while humidity and texture demonstrated that there is no correlation regarding to salt. There were obtained values of humidity from 59,36 to $65,69 \%$ and texture from 11,86 to $20,0 \%$ that permit to characterize the average texture values as good. Taking into account the results obtained in the multiple optimization, it was obtained the best formulation of melted salts for spreadable melted cheese: $70 \%$ of sodium biphosphate and $30 \%$ of sodium monocitrato, or use only $3 \%$ of texture with sodium biphosphate since both formulations offer as a result the desirable texture.

Keywords: spreadable melted cheese, flux salts, sodium biphosphate salts and sodium monocitrato.

\section{Introducción}

La elaboración del queso, junto con el pan, el vino y la cerveza, es uno de los procesos biotecnológicos más antiguos que el hombre ha realizado. Se cree que este producto tiene su origen en la costumbre de pueblos del mediterráneo de transportar la leche en odres hechos con pieles de animales, 0 con estómagos 0 vejigas.

En algún momento se utilizó un estómago fresco, con restos de enzimas coagulantes y el resultado final, sin buscarlo, fue el queso. Durante siglos, la elaboración del queso se ha ido modificando $y$ refinando. Su fabricación se mantuvo como una actividad artesanal hasta la aplicación de las bases científicas que comenzaron a principios del siglo XX, permitiendo la fabricación a gran escala. En la actualidad las variedades de queso más populares se elaboran industrialmente y el queso es un producto muy importante en la dieta de muchos países (Huallpa, 2018; Palatnik, 2019).

Se entiende por el queso procesado y queso fundido para untar (Tipo Americano) los quesos obtenidos por molturación, mezcla, fusión y emulsión con tratamiento térmico y agentes emulsionantes de una o más variedades de queso, con o sin la adición de componentes de leche $\mathrm{u}$ otros productos alimenticios (NEIAL-129, 1978; Sandoval, 2018; Anaya y Salinas, 2020).

El queso procesado o fundido se ha convertido en una buena alternativa para las industrias lácteas, debido a que tiene un período de vida útil prolongado y presenta mejor facilidad de manejo en las empresas. Se obtiene mezclando, agitando y calentando hasta fundir una mezcla de quesos crudos, con sales emulsionantes, agua y limitada 
cantidad de ingredientes adicionales (Arteaga, Hernández y Peñate, 2020; Vaca, 2020).

Las sales emulsionantes son de gran importancia para la elaboración de queso procesado por que tienen un efecto en las propiedades químicas, físicas y microbiológicas al final del producto. Las sales comúnmente más utilizadas para la fabricación de queso procesado incluyen citratos de sodio, ortofosfatos de sodio, pirofosfatos de sodio, tripolifosfatos de sodio, polifosfatos de sodio, fosfatos básicos de sodio o aluminio y mezclas de fosfatos. Estas sales en gen eral deben tener un catión monovalente y un anión polivalente (Hernández, 2018; Becerra, Rey y Carvajal, 2019).

Las sales promueven con ayuda de calor y cizallamiento, una serie de concertados cambios fisicoquímicos en la mezcla de queso que resultan en la rehidratación de la matriz paracaseínica y su conversión en un agente emulsionante activo. Estos cambios incluyen la retención de calcio, ajuste al alza y la estabilización (buffering) de $\mathrm{pH}$, la hidratación de la paracaseína (solvatación), la dispersión-emulsión de grasa, y la formación estructural del queso después del enfriamiento (Hernández, 2018; Huallpa, 2018).

Las sales emulsionantes no actúan directamente como emulsionantes, aumentan el potencial emulsionante de la caseína formando sales complejas solubles y paracaseinato alcalino soluble $(\mathrm{Na}, \mathrm{K})$ a partir de los cationes de la caseína que se hallan disponibles en el queso como paracaseinato de calcio insoluble. Por lo tanto, el paracaseinato alcalino soluble que se obtiene actúa como emulsionante y forma la emulsión ～(Hernández, 2018; Huallpa, 2018).

El papel esencial de las sales fundentes en la elaboración de queso procesado es complementar la capacidad de emulsión de las proteínas del queso.

En este sentido, Yero (2010) establece en sus estudios una proporción adecuada de sales fundentes para lograr una buena calidad en el queso fundido cortable, utilizando como materia prima cuajada fresca fijando el $\mathrm{pH}$ de la misma. Los resultados obtenidos en las determinaciones químico-físicas, así como en la prueba sensorial de perfil de textura al producto 
terminado muestran que para el $3 \%$ (30 \% de monocitrato de sodio y 70 $\%$ bifosfato de sodio) se alcanzaron mejores resultados del grado de satisfacción de las variables respuesta, y de los parámetros de calidad del queso.

Por su parte, Lora (2011) refiere la proporción adecuada de sales fundentes para lograr una buena calidad en el queso fundido cortable partiendo de materias primas con diferentes valores de $\mathrm{pH}$. Los valores obtenidos en la optimización de la mezcla de sales fundentes para las variables respuestas, utilizando el 3 $\%$ de la masa total para la materia prima de $\mathrm{pH}=5,4$ mostraron los mejores resultados para una proporción de $(60,0 \%$, de bifosfato de sodio, $40 \%$ de monocitrato de sodio) mientras que para las corridas de $\mathrm{pH} 5,8$ como materia prima se obtuvo una formulación de $(80 \%$ de bifosfato de sodio y un $20 \%$ de monocitrato de sodio).

Se conoce que para obtener mejores resultados dentro de las producciones de queso fundido untable es preciso emplear sales fundentes de forma combinada, como por ejemplo el caso de las sales comerciales, las cuales son fórmulas cuidadosamente obtenidas en investigaciones. Cada fórmula está destinada a una función específica, en un tipo de queso fundido específico y está compuesta fundamentalmente por mezclas de fosfatos, polifosfatos y citratos en proporciones solo conocidas por los productores (Hernández, 2018; Huallpa, 2018; López, Barriga y Pedregosa, 2018).

En consecuencia, los autores consideran que, al ser la proporción adecuada de estas mezclas de sales fundentes solo conocidas por los productores de queso fundido, puede traer consigo problemas de textura en el producto que se obtiene, afectándose específicamente la untabilidad de este; lo que hace necesario buscar nuevas formulaciones de estas sales fundentes, para mejorar la calidad de las producciones de queso fundido untable.

En concordancia con lo antes expuesto, se plantea como problema de investigación: ¿Cuál es la composición adecuada de una mezcla de sales fundentes para lograr un queso fundido untable con la textura requerida, partiendo de materias primas frescas? 
Por consiguiente, el objetivo de la investigación es establecer una proporción adecuada de sales fundentes que permita alcanzar la textura requerida en el queso fundido untable, mediante una mezcla de sales de bifosfato y monocitrato de sodio.

\section{Metodología}

La investigación fue llevada a cabo en la planta piloto de la Universidad de Camagüey Ignacio Agramonte Loynaz. Se realizaron producciones experimentales de queso fundido untable a escala de laboratorio según el flujo tecnológico que se muestra en la figura 1.

Figura 1. Diagrama de flujo del proceso tecnológico.

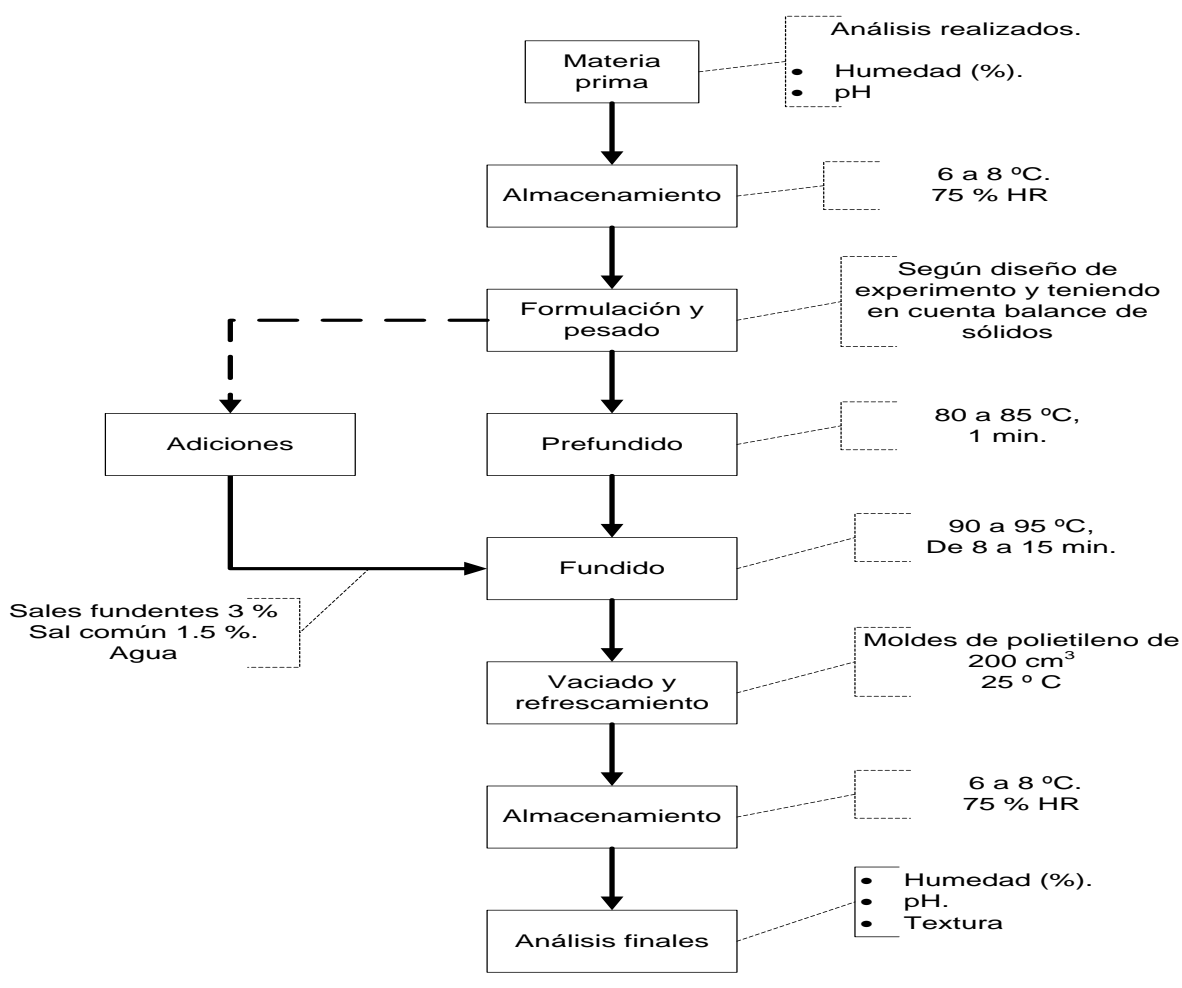

\section{Materias primas}

Las materias primas utilizadas fueron: cuajada fresca, procedentes de la fábrica La Vaquita, la que fue transportada congelada y luego se conservó a una temperatura de 2 a 8 $\stackrel{\circ}{ } \mathrm{C}$; sales fundentes (monocitrato y bifosfato de sodio), agua y sal común (cloruro de sodio).

\section{Ajuste de sólidos totales}

Se realizó una fundición sin la adición de agua con el objetivo de determinar la pérdida de esta durante el proceso, utilizando el 
método gravimétrico. Luego se procedió al ajuste de los sólidos totales del producto mediante la adición de agua. Se determinó la cantidad de cuajada y de agua necesaria en la elaboración del queso fundido untable, teniendo en cuenta la pérdida de la misma en la corrida anterior, el porciento de sólidos totales de la materia prima y el porciento deseado en el producto terminado, partiendo del siguiente balance de masa.

Figura 2. Diagrama de balance de masa del queso fundido.

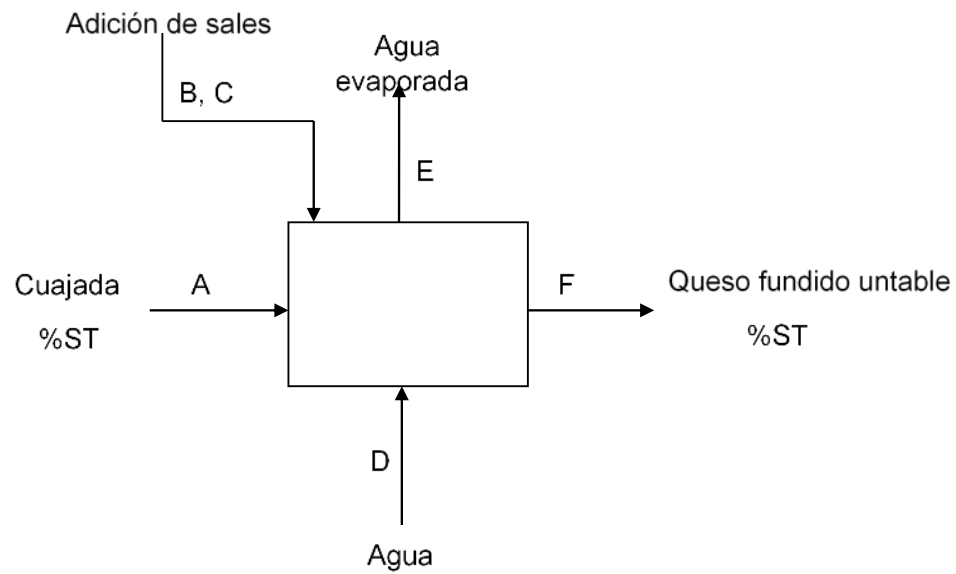

Corrientes presente en el balance de masa:
$A \rightarrow$ Cuajada
$\mathrm{B} \rightarrow$ Sales fundentes
$\mathrm{C} \rightarrow$ Sal común
$\mathrm{D} \rightarrow$ Agua $\quad \mathrm{E} \rightarrow$ Agua evaporada
$F \rightarrow$ Queso fundido

Balance total:

$\sum m(A)+m(B)+m(C)+m(D)=m(E)+m(F)$

Balance ST:

$\% S T * m(A)+\% S T^{*} m(B)+\% S T * m(C)=\% S T * m(F)$

\section{Proceso tecnológico utilizado}

Teniendo en cuenta lo normado en (NEIAL-1609-20,1990), la fabricación del queso fundido constó de los siguientes pasos:

Molinado y triturado del queso materia prima: en esta etapa se molió la cuajada y se le determinó el $\mathrm{pH}$ inicial y el porciento de sólidos totales de la misma. Luego se realizó el pesado del queso molido para determinar la masa a añadir en cada una de las corridas, y también se 
pesaron las diferentes masas de sales fundentes a utilizar en las mismas.

Se mezcló la cuajada con agua, sal común (cloruro de sodio) y sales fundentes (monocitrato y bifosfato de sodio) y se procedió a la fundición de la mezcla a una temperatura de 90 a $95{ }^{\circ} \mathrm{C}$ por un tiempo de 8 a 15 minutos. La misma se llevó a cabo en una fundidora experimental, compuesta por un baño María que alcanzó una temperatura superior a los $100^{\circ} \mathrm{C}$, para la fundición del queso se empleó un envase de aluminio, un termómetro y un agitador mecánico con revoluciones constante para evitar la aparición de partículas quemadas y posibilitar una fundición homogénea.

Luego se pasó al envasado del queso fundido en moldes plásticos el que se realizó a la misma temperatura que salió del fundidor. El refrescamiento de la masa fundida se llevó a cabo a una temperatura ambiente para evitar alteraciones por cambios bruscos de temperatura, por un tiempo de 25 a 30 minutos. Se le realizaron los análisis de $\mathrm{pH}$ y porciento de sólidos totales a los quesos fundidos obtenidos y se almacenaron en neveras de conservación a una temperatura de 6 a $10^{\circ} \mathrm{C}$.

Cada producción se realizó en base a obtener $100 \mathrm{~g}$ de producto terminado. Se utilizó un $3 \%$ de mezcla de sales fundentes (monocitrato y bifosfato de sodio), y $1,5 \%$ de sal común (cloruro de sodio).

\section{Análisis realizados}

Se realizaron las siguientes determinaciones químico-físicas y sensoriales y se compararon con los parámetros de calidad establecido en la (NC-664, 2008).

Determinación de pH, (\%) humedad y determinación de la textura.

Se realizó la evaluación sensorial según el procedimiento analítico establecido para la industria láctea, el cual se basa en la evaluación de los defectos de cada uno de los atributos que conforman cada una de las características organolépticas de un producto determinado, mediante escalas estructuradas de 5 puntos. Se evaluó la textura, por ser de vital importancia en la evaluación de la calidad sensorial del queso fundido untable. 


\section{Diseño de la investigación}

La investigación fue realizada mediante un diseño de mezcla Simplex-Lattice con dos componentes (monocitrato sódico y bifosfato sódico) y 3 variables respuestas ( $\mathrm{pH}$, humedad y textura), para la cual se llevaron a cabo 15 corridas experimentales.

A partir del diseño de mezcla Simplex-Lattice se utilizaron dos modelos estadísticos: El cuadrático y el cúbico. Estos fueron seleccionados en dependencia del valor de probabilidad obtenida en cada modelo. El mismo se comparó con 0,05 asumiendo que se trabaja con un $95 \%$ de confiabilidad, fue seleccionado el que tuvo un valor de probabilidad menor.

\section{Análisis de la mejor formulación}

Se realizaron tres corridas utilizando una materia prima diferente con la mejor formulación obtenida en la optimización de los componentes (70 $\%$ de bifosfato de sodio y $30 \%$ de monocitrato de sodio) y así poder comprobar los resultados estadísticos obtenidos en para $\mathrm{pH}$, (\%) de humedad y textura.
Análisis de la utilización de bifosfato como única sal fundente

Se realizaron tres corridas con bifosfato de sodio como sal fundente en la mezcla para comparar con los resultados utilizando la mejor corrida.

\section{Procesamiento de los resultados}

Los resultados obtenidos se procesaron mediante la utilización del programa Microsoft Excel y el paquete estadístico Statgraphics Centurion XV (StatPointInc., 2006), para obtener una mezcla óptima que garantice la mejor calidad del queso fundido untable.

\section{Resultados}

\section{Materias primas a utilizar}

Para la elaboración del queso fundido untable se utilizó como materia prima: cuajada producida en la fábrica La Vaquita. La misma se mantuvo en conservación a una temperatura de 2 a $8{ }^{\circ} \mathrm{C}$ por un período de 3 días. Se emplearon las sales fundentes bifosfato de sodio y monocitrato de sodio de $\mathrm{pH} 8,95 \mathrm{y}$ 9,65 respectivamente, sal común (cloruro de sodio) y agua. 
En la tabla 1. se muestran los parámetros fisicoquímicos de las materias primas utilizadas en la fabricación del queso fundido untable.

Tabla 1. Parámetros físico-químicos de las materias primas utilizadas.

\begin{tabular}{|c|c|c|c|}
\hline Materias primas & $\mathbf{p H}$ & Humedad (\%) \\
\hline Cuajada & & 5,47 & 42,2 \\
\hline Bifosfato de sodio & & 8,95 & 0,1 \\
\hline Monocitrato de sodio & & 9,65 & 0,1 \\
\hline
\end{tabular}

Fuente: Autores (2021).

Evaluación de la formulación de

las sales para queso fundido untable.

Para la evaluación en la formulación se realizaron 15 corridas experimentales con diferentes proporciones de las sales fundentes (monocitrato y bifosfato sódico), utilizando cuajada fresca como materia prima.

Tabla 2. Resumen estadístico para el $\mathrm{pH}$, (\%) de humedad y textura.

\begin{tabular}{|c|c|c|c|}
\hline & $\begin{array}{c}\text { Efecto de la } \\
\text { evaluación en la } \\
\text { formulación de las } \\
\text { sales fundentes } \\
\text { sobre el pH }\end{array}$ & $\begin{array}{c}\text { Efecto de la } \\
\text { formulación de las } \\
\text { sales fundentes } \\
\text { sobre la humedad } \\
(\%)\end{array}$ & $\begin{array}{c}\text { Efecto de la } \\
\text { formulación de las } \\
\text { sales sobre la } \\
\text { textura }\end{array}$ \\
\hline Número corridas & 15 & 15 & 15 \\
\hline Media & 6,28 & 62,5 & 17,9 \\
\hline $\begin{array}{c}\text { Coeficiente de } \\
\text { variación }\end{array}$ & $1,32 \%$ & $2,83 \%$ & $13,76 \%$ \\
\hline Varianza & 0,006 & 3,14 & 6,09 \\
\hline $\begin{array}{c}\text { Desviación } \\
\text { estándar }\end{array}$ & 0,07 & 1,77 & 2,46 \\
\hline Mínimo & 6,14 & 59,36 & 11,86 \\
\hline Máximo & 6,41 & 65,59 & 20,0 \\
\hline
\end{tabular}

Fuente: Autores (2021).

Efecto de la evaluación en la formulación de las sales fundentes sobre el $\mathrm{pH}$

Los resultados obtenidos en el procesamiento estadístico para los valores de $\mathrm{pH}$ se muestran en la tabla 2; en el que se puede observar un coeficiente de variación $1,32 \%$, el cual demuestra que existe una variabilidad mínima entre los valores de $\mathrm{pH}$ del queso fundido en cada una de las corridas realizadas. 
Dentro de los resultados obtenidos muestran una media para el $\mathrm{pH}$ de 6,28 el que se encuentra ligeramente desplazado de los parámetros establecidos en los documentos normativos para quesos fundidos (NC-664, 2008). Esto se debe al empleo de sales básicas, las cuales ajustan el $\mathrm{pH}$ del producto final sobre lo básico, además de utilizar un queso materia prima con un $\mathrm{pH}$ no muy ácido, por lo que para lograr obtener un $\mathrm{pH}$ final que se encuentre dentro de los parámetros establecidos debe de utilizarse un regulador de la acidez como el ácido cítrico.

La modelación para la variable $\mathrm{pH}$, mostró que existe ajuste entre la variación de la formulación de la mezcla y la variación de los valores de $\mathrm{pH}$ obtenidos en los resultados. Esto pudo comprobarse con los valores de R2 59,31\% y R2 ajustado $48,22 \%$ alcanzados, los que demuestran que hay relativamente buen grado de ajuste en el modelo utilizado. Además, el valor de probabilidad fue de $(0,01)$ el cual es menor que 0,05 por lo que evidencia que hay una significativa variación del pH en relación con la variación de los componentes (bifosfato y monocitrato de sodio).

Para las fundiciones realizadas se estima que se alcanzó un buen grado de ajuste. Esto confirma que el $\mathrm{pH}$ que se obtiene en el producto final guarda estrecha relación con las proporciones de sales fundentes, debido a que la adición de estas se realiza en función del $\mathrm{pH}$ de las materias primas, con el objetivo de estabilizar el $\mathrm{pH}$ del producto final. Por tanto, las proporciones de las sales fundentes ejercen una acción directa sobre este parámetro.

\section{Efecto de la formulación de las} sales fundentes sobre la humedad

Los resultados logrados en el procesamiento estadístico para los valores de humedad se muestran en la tabla 2. La media obtenida fue de $62,5 \%$ de humedad, encontrándose desplazada de los parámetros establecidos para la producción de queso fundido untable (NC-664, 2008). El coeficiente de variación fue de $2,81 \%$ por lo que no hay grandes diferencias entre los valores de humedad obtenidos.

La modelación de la correlación para la variable humedad mostraron que 
hay poca correlación entre la variación de la formulación de la mezcla de sales fundentes y la variación de humedad obtenida. Esto se observó en los valores de $\mathrm{R} 2=5,01 \%$ y $\mathrm{R} 2$ ajustado $0,0 \%$ alcanzados, por lo que existe poca o ninguna relación entre ambas variables, debido a que las sales fundentes no tienen influencia significativa en el contenido de sólidos de la mezcla. Solamente interfieren en la homogeneidad de la masa obtenida en el producto.

La inestabilidad de la humedad del producto final se debe a la etapa de fundido, esta se trató de realizar lo más estándar posible, pero al realizar la operación en una fundidora artesanal no se pudo lograr este objetivo, ya que no se alcanza una temperatura constante lo que provoca que en unos procesos se evapore más agua que en otros, además de no contarse con un equipo para crear vacío.

\section{Efecto de la formulación de las sales sobre la textura}

Los resultados logrados en el procesamiento estadístico para los valores de textura se muestran en la tabla 2. Los resultados obtenidos en el procesamiento estadístico realizado para la evaluación de la textura muestran como varió la puntuación del queso fundido untable en algunas de las corridas.

En los resultados alcanzados la puntuación media fue de 17,9 encontrándose dentro del rango de puntuación entre $(17-17,9)$ por lo que se alcanza una evaluación de buena. Algunas de estas muestras presentaron algunos defectos desde ligeros hasta marcados en cuanto a la textura. Un ejemplo de las muestras afectadas fueron la 3 con (30\% de monocitrato de sodio y $70 \%$ de bifosfato de sodio), que presentó grumos y oquedades de forma ligera y masa dura de forma marcada; y la muestra 9 con $(20 \%$ de monocitrato de sodio y $80 \%$ de bifosfato de sodio) la que presentó una ligera presencia de grumo y masa moderadamente dura. El coeficiente de variación fue de 13,76 \% aproximadamente, lo que demostró que hay diferencia entre los valores obtenidos para cada muestra.

Según los resultados obtenidos se evidenció poca correlación entre la variación de la formulación de la mezcla y la variación de la textura. Esto se pudo comprobar con los 
valores de R2 13,51\% y R2 ajustado $0,0 \%$ alcanzados, los que fueron insignificantes.

Optimización de la mezcla para queso fundido untable

Para la optimización de la mezcla se determinó la combinación de los componentes experimentales (bifosfato y monocitrato de sodio) con la optimización simultánea de las variables respuestas $\mathrm{pH}, \quad(\%)$ humedad y textura. En la optimización para los valores de $\mathrm{pH}$ se obtuvo una optimización de 6,28 relativamente alto con respecto a lo normado en (NC-664, 2008), este resultado se debe a la influencia del $\mathrm{pH}$ de las sales fundentes bifosfato $\mathrm{y}$ monocitrato de sodio $(8,95$ y 9,65$)$, las cuales presentan un fuerte poder alcalino. Para la modelación usada, el nivel significativo fue de 0,05 y los valores finales a alcanzar para cada uno en la optimización de las variables fueron los máximos ( $\mathrm{pH}$ de 6,28; sólidos totales de 62,66 y textura de 17,33 ).

Los resultados de la optimización realizada para la concentración de las sales monocitrato de sodio y bifosfato de sodio: bifosfato $69,54 \mathrm{y}$ monocitrato 30,45 muestran que se alcanzaron teniendo en cuenta la optimización de las variables respuestas.

Los valores obtenidos en la optimización de la mezcla de sales fundentes evidenciaron una proporción de aproximadamente 70 $\%$ de bifosfato de sodio y $30 \%$ de monocitrato de sodio lo que correspondió a la hipótesis planteada para la investigación.

Al comparar estos resultados con los alcanzados por Yero (2010) se evidencia que la optimización de la mezcla total es prácticamente igual. Mientras que estableciendo la comparación con los resultados obtenidos por Lora (2011) se muestra un aumento del porciento del bifosfato de sodio con respecto a la mezcla total, debido a que el bifosfato es el más usado sobre todo cuando el pH del queso está comprendido entre 6,0 y 6,3 .

Por tanto, al emplear una materia prima con pH 5,4 se debe utilizar una mayor proporción posible de bifosfato de sodio para evitar que el $\mathrm{pH}$ final del producto a obtener sea mayor que el establecido para queso fundido untable. 
Evaluación en el efecto de la mejor formulación y el empleo de una sal única en la mezcla

Para la elaboración del queso fundido untable se utilizó queso como materia prima, a la cual se le determinaron los parámetros físicoquímico siguientes: $\mathrm{pH} \quad 5,97$ y humedad $45,8 \%$.

Tabla 3. Resultados estadísticos para el pH y la humedad.

\begin{tabular}{|c|c|c|c|c|}
\hline & $\begin{array}{c}\text { Número } \\
\text { de } \\
\text { corridas }\end{array}$ & Media & $\begin{array}{c}\text { Coeficiente } \\
\text { de } \\
\text { variación }\end{array}$ & $\begin{array}{c}\text { Desviación } \\
\text { estándar }\end{array}$ \\
\hline $\begin{array}{c}\text { Efecto de } \\
\text { la mejor } \\
\text { formulación } \\
\text { sobre el pH }\end{array}$ & 3 & 6,56 & 0,35 & 0,02 \\
\hline $\begin{array}{c}\text { Efecto de } \\
\text { la mejor } \\
\text { formulación } \\
\text { sobre la } \\
\text { humedad }\end{array}$ & 3 & 62,5 & 2,49 & 1,55 \\
\hline
\end{tabular}

Fuente: Autores (2021).

\section{Efecto de la mejor formulación sobre el pH}

Los resultados logrados en el procesamiento estadístico para los valores de $\mathrm{pH}$ se muestran en la tabla 3. Se puede observar un coeficiente de variación $0,35 \%$, el que demuestra que existe una mínima variabilidad entre los valores de $\mathrm{pH}$ del queso fundido untable en las corridas realizadas.

Los resultados obtenidos muestran una media de 6,56 la cual se encuentra desplazada totalmente de los parámetros establecidos según
(NC-664, 2008) para el queso fundido untable. Esto se debe al empleo del queso materia prima el que contaba con un $\mathrm{pH}$ alto.

Teniendo en cuenta que los valores de $\mathrm{pH}$ obtenido en los resultados de los análisis estadísticos realizados a las muestras de queso fundido se encontraban fuera de lo normado (NC-664, 2008) se hace necesario el empleo de reguladores de $\mathrm{pH}$ como el ácido cítrico, ácido láctico o el uso de suero ácido en sustitución del agua para disminuir el $\mathrm{pH}$ del producto final y así lograr que éste 
entre los límites establecidos en los documentos normativos.

\section{Efecto de la mejor formulación sobre la humedad}

Los resultados logrados en el procesamiento estadístico para los valores de humedad se muestran en la tabla 3. La media obtenida fue de $62,5 \%$, igualmente que la obtenida en las corridas anteriores. Estas se encontraron desplazadas de los parámetros establecidos según lo normado para la producción de queso fundido untable (NC-664, 2008).

El coeficiente de variación obtenido en los resultados estadísticos es de $2,49 \%$ lo que muestra que hay poca variabilidad en los valores de humedad obtenidos por lo que se evidencia el buen grado de ajuste en el modelo utilizado para la humedad.

\section{Efecto de la mejor formulación sobre la textura}

Según las corridas que se llevaron a cabo con la mejor formulación mostraron una puntuación perfecta, donde las tres corridas realizadas alcanzaron la máxima puntuación. Esta evaluación obtenida corrobora que la mejor proporción a utilizar en la formulación del queso fundido untable es la de $(70 \%$ de bifosfato de sodio y $30 \%$ de monocitrato de sodio).

Análisis de la utilización de bifosfato de sodio como única sal fundente. Efecto del bifosfato sobre el pH

Tabla 4. Resultados estadísticos para el pH, la humedad y la textura.

\begin{tabular}{|c|c|c|c|c|}
\hline & $\begin{array}{c}\text { Número } \\
\text { de } \\
\text { corridas }\end{array}$ & Media & $\begin{array}{c}\text { Coeficiente } \\
\text { de } \\
\text { variación }\end{array}$ & $\begin{array}{c}\text { Desviación } \\
\text { estándar }\end{array}$ \\
\hline $\begin{array}{c}\text { Efecto del } \\
\text { bifosfato sobre } \\
\text { el pH }\end{array}$ & 3 & 6,65 & 0,75 & 0,05 \\
\hline $\begin{array}{c}\text { Efecto del } \\
\text { bifosfato sobre } \\
\text { la humedad }\end{array}$ & 3 & 62,75 & 0,92 & 0,57 \\
\hline $\begin{array}{c}\text { Efecto de la } \\
\text { evaluación del } \\
\text { bifosfato sobre } \\
\text { la textura }\end{array}$ & 3 & 19,54 & 2,30 & 0,45 \\
\hline
\end{tabular}

Fuente: Autores (2021). 


\section{Efecto del bifosfato sobre el pH}

Los resultados logrados en el procesamiento estadístico para los valores de $\mathrm{pH}$ se muestran en la tabla 4. Se observa un coeficiente de variación de 0,75 lo que hay una variabilidad mínima entre los valores de $\mathrm{pH}$ obtenidos.

En los resultados estadísticos se muestra una media de 6,65 que se encuentra por encima de los parámetros establecidos en los documentos normativos para el queso fundido (NC-664, 2008), estos valores de $\mathrm{pH}$ se encuentran desplazados debido al empleo de un queso materia prima elaborado que poseía un alto $\mathrm{pH}$, por lo que para que el $\mathrm{pH}$ se encuentre dentro de los parámetros es necesario el empleo de algún regulador.

\section{Efecto del bifosfato sobre la humedad}

Los resultados logrados en el procesamiento estadístico para los valores de humedad se muestran en la tabla 3. El coeficiente de variación alcanzado fue de $0,92 \%$ el cual muestra que existe muy poca variabilidad en los resultados de humedad obtenidos. La media fue de
$62,75 \%$ y al igual que las anteriores se encuentra desplazada ligeramente de los parámetros establecidos (NC-664, 2008) para el queso fundido, debido a la utilización de la fundidora artesanal, la cual no alcanza una temperatura constante.

Efecto de la evaluación del bifosfato sobre la textura

Los resultados logrados en el procesamiento estadístico para los valores de textura se muestran en la tabla 3. Los resultados obtenidos en procesamiento estadístico realizado para la evaluación de la textura muestran un coeficiente de variación de 2,30 \% el cual prueba la poca variación de la puntuación del queso fundido untable en las corridas realizadas.

En los resultados alcanzados la puntuación media fue de 19,54 encontrándose dentro del rango de puntuación (19-20) alcanzando evaluación de excelente.

\section{Conclusiones}

Los resultados obtenidos del procesamiento estadístico para la variable $\mathrm{pH}$, muestran buena correlación ya que los valores son 
elevados, pero el valor de $\mathrm{pH}$ que se obtuvo está por encima de lo normado, debido al alto valor de $\mathrm{pH}$ de las materias primas. Según los resultados obtenidos del procesamiento estadístico para las variables humedad y textura, no existe correlación con respecto a las sales. Se obtuvo valores de humedad de 59,36 a 65,69 \% y la textura de 11,86 a 20,0 siendo la media de los valores de textura evaluados como bueno. Atendiendo a los resultados alcanzados en la optimización múltiple se logró que la mejor formulación de sales fundentes para queso fundido untable es: $70 \%$ de bifosfato de sodio y $30 \%$ de monocitrato de sodio, o emplear el $3 \%$ de la mezcla solo con bifosfato de sodio ya que ambas formulaciones ofrecen como resultado la textura deseada.

\section{Bibliografía}

Anaya, G. P. \& Salinas, C. E. (2020). Aprovechamiento del suero de queso fresco en la elaboración de queso crema untable enriquecido con sólidos proteicos. Tesis de maestría no publicada, Universidad Nacional del Callao, Callao, Perú. Recuperado de http://repositorio.unac.edu.pe/ handle/UNAC/5639

Arteaga, M. R., Hernández, H. L. \& Peñate, C. D. (2020). Elaboración de un queso procesado tipo untable obtenido a partir de queso costeño. Información Tecnológica, 31 (12), 187194. ISSN-e: 0718-0764. Recuperado de http://dx.doi.org/10.4067/S07 18-07642020000200187

Becerra, A. C., Rey, J.P. \& Carvajal, Y. M. (2019). Propuesta para el desarrollo de un queso untable de soya saborizado con albahaca. Ingeniería de Alimentos, Universidad Nacional Abierta y a Distancia - UNAD, Duitama, Colombia. Recuperado de https://repository.unad.edu.co /bitstream/handle/10596/2769 2/acbecerrav.pdf?sequence $=$ 1\&isAllowed=y

Cuba, Comité Estatal de Normalización. (1978).

NEIAL-129. Queso fundido. Especificaciones de calidad. La Habana, Cuba: MINAL.

Cuba, Comité Estatal de Normalización. (1990). NEIAL-1609-20. Leche y productos lácteos. Queso fundido. Proceso tecnológico. La Habana, Cuba: MINAL.

Cuba, Comité Estatal de Normalización. (2008). NC664. Queso Fundido. 
Especificaciones de calidad. La Habana, Cuba: CEN

Hernández, L. E. (2018). Elaboración de queso procesado cortable utilizando tres tipos de cuajadas ácidas refrigeradas. Ingeniería Agroindustrial, Universidad Técnica del Norte, Ibarra, Ecuador. Recuperado de http://repositorio.utn.edu.ec/h andle/123456789/7887

Huallpa, G. R. (2018). Elaboración de queso fundido para la empresa sociedad agroindustrial Chonchocoro Limitada (SACH. LTDA.). Licenciatura en Química Industrial Tecnología de Alimentos, Universidad Mayor de San Andrés, La Paz, Bolivia. Recuperado de https://repositorio.umsa.bo/x mlui/handle/123456789/1674 6

López, A.L., Barriga, D. \& Pedregosa, A. (2018). Recomendaciones en la Elaboración de Queso Fundido para untar. Consejería de Agricultura y Pesca, Instituto de Investigación y Formación Agraria y Pesquera, Andalucía, España. Recuperado de https://www.juntadeandalucia .es/agriculturaypesca/ifapa/se rvifapa/registroservifapa/31c78d9c-d4c94689-8a01-38c5bf59b051
Lora, R. (2011). Determinación de proporciones de sales fundentes para queso fundido cortable. Licenciatura en Ciencias Alimentarias, Universidad de Camagüey, Camagüey, Cuba.

Palatnik, D. R. (2019). Desarrollo de quesos funcionales $y$ aprovechamiento de proteínas de lactosuero. Tesis doctoral no publicada, Universidad Nacional de la Plata, La Plata, Argentina. Recuperado de http://sedici.unlp.edu.ar/handl e/10915/87205

Sandoval, J. H. (2018). Evaluación de los riesgos físico-químicos y microbiológicos en la producción de quesos saborizados en la Cooperativa de producción Agropecuaria Chone Ltda. Tesis de maestría no publicada, Escola Superior de Turismo e Tecnología do Mar, Instituto Politécnico de Leiria, Leiria, Portugal. Recuperado de

https://iconline.ipleiria.pt/hand le/10400.8/3477

Vaca, M. E. (2020). Diseño de un queso crema fermentado bajo en grasa. Química de los Alimentos, Universidad Central del Ecuador, Quito, Ecuador. Recuperado de http://www.dspace.uce.edu.e c/handle/25000/21970 